\title{
Medición de la discapacidad vocal en los pacientes con disfonías funcionales
}

\author{
Measurement of the vocal handicap in patients with functional dysphonia \\ Wasim Elhendi $\mathbf{H}^{\mathbf{1}}$, Antonio Caravaca $\mathbf{G}^{\mathbf{1}}$, Sofía Santos $\mathbf{P}^{\mathbf{1}}$.
}

\begin{abstract}
RESUMEN
Introducción: La medición de la discapacidad vocal en los pacientes con disfonía funcional es un tema de gran relevancia.

Objetivo: Analizar los resultados de dos medidas subjetivas (el índice de discapacidad vocal (VHI) y el índice de calidad de vida (QLI)) y calcular el grado de discapacidad que suponen las disfonías funcionales para los pacientes.

Material y método: Sesenta y cinco pacientes diagnosticados de disfonías funcionales contestaron un cuestionario que incluye el índice de discapacidad vocal y el índice de calidad de vida.

Resultados: Encontramos un grado importante de discapacidad reflejado por unos valores elevados del VHI $(55,74)$, sus tres subescalas (orgánica $(24,82)$, funcional $(19,28)$ y emocional $(11,65)$ y del QLI $(11,42)$.

Discusión: Se realiza una revisión exhaustiva de la literatura, constatando la escasez de estudios sobre la discapacidad que producen los trastronos vocales y se comparan nuestros resultados con los de otros autores.

Conclusión: La presencia de disfonías funcionales supone una discapacidad importante a nivel de las actividades sociales y laborales del paciente y un impacto emocional considerable.
\end{abstract}

Palabras clave: Medición; discapacidad vocal; disfonías funcionales.

\section{ABSTRACT}

Introduction: Functional dysphonia is a major issue.

Aim: To analyse the results of two subjective measures (Vocal Handicap Index (VHI) and Quality Life Index (QLI)) and to calculate the impact that functional dysphonias suppose for the patients.

Material and Method: 65 patients diagnosed of functional dysphonia answered a questionnaire that includes the Vocal Handicap Index and the Quality Life Index.

Results: We found an important degree of handicap indicated by high values of the $\mathrm{VHI}(55,74)$, of its three subscales (organic $(24,82)$, functional $(19,28)$ and emotional $(11,65))$ and of the QLI $(11,42)$.

Médico Otorrinolaringólogo. Hospital Punta Europa, Algeciras Cádiz, España. 
Discussion: We made a wide literature review, noting that there are a few studies of the handicap of voice disorders; and compared our results with those of other authors.

Conclusion: The presence of functional dysphonias supposes an important level of handicap in the patient's social and laboral activities and a considerable emotional impact.

Key words: Measurement; vocal handicap; functional dysphonia.

\section{INTRODUCCIÓN}

Los mayores avances en los conocimientos y cuidados de la voz en los últimos años han hecho que se preste mayor atención a dos campos que están emergiendo en la valoración de la voz: el primero de ellos hace referencia a la valoración subjetiva que hace el propio paciente de su voz ya que se considera interesante conocer su opinión que, al final, tendrá mucho que ver con el éxito terapéutico, y por otra parte, los métodos que podemos utilizar para cuantificar la minusvalía o la discapacidad funcional que se puede atribuir a un trastorno vocal en un paciente determinado ${ }^{1}$.

Antiguamente, el estudio del impacto del problema vocal en la calidad de vida del paciente era limitado por la escasez de medios disponibles, pero ahora, y aunque la medición de la discapacidad en las alteraciones vocales está en sus inicios, este tipo de estudios se considera de gran valor.

Es lógico que el impacto de una mínima variación en el tono de un profesional de la voz va a ser mucho mayor que en una persona con demandas vocales mínimas. Así que una lesión laríngea pequeña puede impedir a un profesional de voz desarrollar su profesión habitual, y en este caso el impacto en su calidad de vida va ser importante y pobremente representado por las pruebas objetivas ${ }^{1}$.

Se está investigando para crear un instrumento de valoración de la discapacidad vocal que sea válido, fiable y capaz de valorar la gravedad inicial para una amplia gama de trastornos, y que debe enfrentar a variables específicas que afectan a personas con distintas demandas vocales (por ejemplo, las personas mayores se preocupan por el volumen de su voz, los maestros se preocupan por la duración de su voz y los cantantes temen por la calidad de su voz) ${ }^{2}$.

Uno de los objetivos más importantes a la hora de atender un trastorno vocal es el de establecer el nivel de discapacidad inicial que produce, un pro- blema que carece de regulaciones jurídicas en la actualidad y que puede ser difícil debido al número amplio de áreas afectadas (emocionales, físicas, funcionales, económicas...); y que se puede conseguir a través de una historia clínica detallada, examen físico y, sobre todo, el cálculo de los índices de calidad de vida (totales y específicos para la voz), como son el SF-36, el VHI, el QLI3 .

Además, aunque tanto las mediciones objetivas como la valoración perceptual de las características de la voz proporcionan algo de información sobre la gravedad del trastorno vocal, esas mediciones no proporcionan información sobre la percepción que tiene el propio paciente de la gravedad de su problema vocal ni sobre qué pacientes con trastornos vocales similares experimentan grados diferentes de discapacidad ${ }^{4}$.

Ante la posibilidad de que se solicite al especialista de voz la valoración del grado de discapacidad de un paciente con trastorno vocal, una valoración que puede tener repercusiones médico-legales y que hay que realizarla de una manera justa $y$ razonable, es necesario conocer los métodos que han sido desarrollados con este fin.

Uno de los métodos de valoración subjetiva de la discapacidad vocal más utilizados en la actualidad es el Índice de Discapacidad Vocal (Voice Handicap Index o VHI), propuesto por Jacobson, en 1998, y que consta de un formulario de autoevaluación que explora tres dominios (funcional, orgánico y emocional), mediante 10 preguntas en cada uno (seleccionadas a partir de informes de enfermos, para asegurar que la escala tenga contenido y validez nominal). Las tres subescalas exploradas son: la funcional que describe el efecto del trastorno de la voz en las actividades cotidianas del paciente, la orgánica que valora la percepción del paciente de las molestias laríngeas 0 de las características de la fonación y la emocional que indica la respuesta afectiva del paciente al trastorno vocal. Este test es válido para todos los tipos de alteraciones vocales $y$ ha sido validado estadísticamente ${ }^{5}$. 
Otras medidas utilizadas para este fin son: VOS (Voice Outcome Survey), V-RQOL (Voice Related Quality of Life), VoiSS (Voice Symptoms Scale) y QLI (Quality Life Index).

Las Guías para la Evaluación de la Incapacidad Permanente de la Asociación Médica Americana (AMA) en lo que respecta a la voz no están lo suficientemente desarrolladas, ya que consideran los problemas de la voz y del habla como un problema único, cuando en realidad son dos subespecialidades que se han desarrollado de forma independiente como dos aspectos distintos de la comunicación verbal y, en general, los criterios de la AMA subestiman los problemas de la voz. No obstante, es innegable que una persona con una voz deteriorada puede perder eficiencia en sus funciones sociales y laborales, y tener graves problemas de comunicación oral y frustración.

\section{OBJETIVO}

El objetivo de este trabajo es analizar los resultados de dos medidas subjetivas. El índice de discapacidad vocal (VHI) y el índice de calidad de vida (QLI) y calcular el grado de discapacidad que supone el trastorno vocal para los pacientes con disfonías funcionales.

\section{MATERIAL Y MÉTODO}

Hemos realizado el estudio sobre una muestra de 65 pacientes disfónicos diagnosticados de disfonía funcional, con edad media de 34 años (mínimo de 13 y máximo de 59), de los cuales 57 eran mujeres; remitidos a la Unidad de Foniatría de nuestro servicio, desde las consultas básicas de Otorrinolaringología, en un periodo de cinco años.

Todos los pacientes rellenaron una encuesta diseñada para describir su propia valoración subjetiva del trastorno vocal que presentan, antes de iniciar cualquier tratamiento. Dicha encuesta se diseñó basándonos en:

1. Medida del índice de la discapacidad vocal (VHI) de Jacobson y cols., introduciendo pequeñas modificaciones lingüísticas para adaptarla a nuestro medio. El paciente tenía que marcar un número que corresponde con la frecuencia con que presenta las situaciones propuestas, donde: 0 significa "Nunca", 1: "Casi nunca", 2: "A veces", 3. "Casi Siempre" y 4: "Siempre". Calculamos la puntuación que corresponde a cada subescala por separado y la puntuación total del test. La encuesta que hemos empleado se representa en la Tabla 1.

2. Medida del índice de calidad de vida (Quality Life Index: QLI), propuesto por Wilson y cols. y que evalúa, fundamentalmente, la severidad de la sintomatología asociada al trastorno vocal y dónde se considera que una puntuación mayor de 5 indica algún grado de deterioro de la calidad de vida asociado al trastorno vocal ${ }^{6}$. Se expone en la Tabla 2.

Los procedimientos de selección y estudio de los pacientes satisfacen las normas éticas establecidas por un comité de ética de nuestra institución y que son concordantes con la declaración de Helsinski (1975).

\section{RESULTADOS}

En la Tabla 3 se resumen los datos obtenidos. Para cada subescala, para el índice de discapacidad vocal total y para el índice de calidad de vida se calcula la media, la desviación típica, la mediana, el valor mínimo, el valor máximo, el intervalo de confianza al $95 \%$ y su error estándar.

\section{DISCUSIÓN}

En nuestro estudio encontramos que las disfonías funcionales suponen un grado de discapacidad importante para la mayoría de los pacientes tanto en el aspecto físico como en el funcional y el emocional (con puntuaciones bastante elevadas) y que la subescala más afectada es la orgánica seguida por la funcional, mientras el aspecto emocional fue el que menos percutía sobre la calidad de vida de estos pacientes. En cuanto al índice de calidad de vida, encontramos una afectación considerable, lo que indica la presencia, en un número elevado de pacientes de sintomas asociados al trastorno vocal (como por ejemplo: tos, carraspeo, sequedad de boca, odinofagia...), lo que empeora aún más su calidad de vida 6 .

La patología vocal es, en su mayor parte, discapacitante para un buen número de pacientes, 
Tabla 1. Cuestionario para el cálculo del Índice de Discapacidad Vocal

Parte I ó $\mathrm{F}$ (Subescala funcional)

1 La gente oye con dificultad mi voz:

2 La gente me entiende con dificultad en sitios ruidosos:

3 Mi familia no me oye si la llamo desde el otro lado de la casa:

4 Uso el teléfono menos de lo que desearía:

5 Tiendo a evitar la conversación en grupo debido a mi voz:

6 Hablo menos con mis amigos y familiares debido a mi voz:

7 La gente me pide que repita lo que digo al hablar cara a cara:

8 Mis problemas con la voz alteran mi vida personal y social:

9 Me siento desplazado de las conversaciones por mi voz:

10 Mi problema de voz me hace perder dinero:

Parte II ó 0 (Subescala orgánica)

11 Noto que pierdo aire por la boca cuando hablo:

12 Mi voz suena diferente a lo largo del día:

13 La gente me pregunta "¿Qué te pasa con la voz?":

14 Mi voz suena ronca y seca:

15 Siento que necesito tensar la garganta para producir la voz:

16 Nunca sé como va a ser mi voz cuando voy a hablar:

17 Trato de cambiar mi voz para que suene mejor:

18 Me esfuerzo mucho para hablar:

19 Mi voz empeora por la tarde:

20 Mi voz se altera, o "se me va" en mitad de una frase:

Parte III ó E (Subescala emocional)

21 Estoy tenso cuando hablo con los demás debido a mi voz:

22 La gente parece irritada por mi voz:

23 Creo que la gente no comprende mi problema de voz:

24 Mi voz me molesta:

25 Progreso menos debido a mi voz:

26 Mi voz me hace sentir discapacitado:

27 Me siento molesto cuando me piden que repita una frase:

28 Me siento avergonzado cuando me piden repetir una frase:

29 Mi voz me hace sentir incompetente:

30 Estoy avergonzado de mi problema con la voz:

$\begin{array}{lllll}0 & 1 & 2 & 3 & 4 \\ 0 & 1 & 2 & 3 & 4 \\ 0 & 1 & 2 & 3 & 4 \\ 0 & 1 & 2 & 3 & 4 \\ 0 & 1 & 2 & 3 & 4 \\ 0 & 1 & 2 & 3 & 4 \\ 0 & 1 & 2 & 3 & 4 \\ 0 & 1 & 2 & 3 & 4 \\ 0 & 1 & 2 & 3 & 4 \\ 0 & 1 & 2 & 3 & 4\end{array}$

Tabla 2. Cuestionario para el cálculo del Índice de Calidad de Vida

\begin{tabular}{|c|c|c|c|c|c|c|}
\hline Síntoma & \multicolumn{3}{|c|}{ Nunca } & & \multicolumn{2}{|c|}{ Siempre } \\
\hline - Necesidad de aclarar la garganta antes de hablar: & 0 & 1 & 2 & 3 & 4 & 5 \\
\hline - Hablo menos con la gente debido a mi problema con la voz & 0 & 1 & 2 & 3 & 4 & \\
\hline - Presencia de molestias (o del dolor) de garganta que interfieren en & 0 & 1 & ? & 2 & & \\
\hline - MI trabajo o mis activlaades alarlas. & 0 & 1 & 2 & 3 & 4 & 5 \\
\hline - Presencia de problemas respiratorios que interfieren con mi trabajo & 0 & 1 & 2 & 3 & 4 & 5 \\
\hline o mis actividades diarias: & 0 & 1 & 2 & 3 & 4 & 5 \\
\hline - Problemas de deglución (sólidos o líquidos): & 0 & 1 & 2 & 3 & 4 & 5 \\
\hline
\end{tabular}


Tabla 3. Análisis estadístico de los resultados del índice de discapacidad vocal, sus tres subescalas y del índice de calidad de vida en los pacientes con disfonías funcionales

\begin{tabular}{|lccccc|}
\hline & & \multicolumn{2}{c|}{ VHI } & QLI \\
\hline Medida & Total & Funcional F & Orgánica 0 & Emocional E & \\
\hline Media & 55,74 & 19,28 & 24,82 & 11,65 & 11,42 \\
Mediana & 55 & 19 & 25 & 10 & 11 \\
Desviación típica & 17,68 & 7,32 & 5,86 & 7,13 & 5,33 \\
Mínimo & 5 & 0 & 5 & 0 & 0 \\
Máximo & 98 & 38 & 36 & 32 & 24 \\
Intervalo de confianza & & & & & \\
al 95\% & $51,36-60,12$ & $17,46-21,09$ & $23,36-26,27$ & $9,88-11,65$ & $10,10-12,76$ \\
Error estándar & 2,19 & 0,91 & 0,73 & 0,88 & 0,66 \\
\hline
\end{tabular}

muchos de los cuales son profesionales de la voz. Estos trastornos cobran su máxima importancia cuando el propio paciente los concibe como la alteración de un parámetro vital que le causa sufrimiento 0 incapacidad para adaptarse a su medio social 0 laboral. Esto es, en definitiva, lo que le hace acudir al terapeuta de voz. Por ello creemos que es importante conocer no sólo las entidades patológicas y las posibilidades de su diagnóstico y tratamiento, sino también, sobre todo en el caso de una patología crónica, contar con elementos de juicio para valorar el grado de discapacidad que suponen ${ }^{1,7}$.

Verdolini y Ramig afirman que aproximadamente 28.000.000 trabajadores en los EE.UU. experimentan problemas diarios de la voz y muchos de ellos refieren que sus problemas vocales les producen un impacto negativo en su trabajo y en su calidad de vida. Las estimaciones basadas en datos empíricos sugieren que, considerando solamente días del trabajo perdidos y costes del tratamiento, el coste social de los problemas de la voz, exclusivamente en los profesores, puede estar cerca de dos mil quinientos millones de dólares anuales en los EE.UU.

Smith y cols. compararon 40 pacientes disfónicos con 200 sujetos sanos pero con problemas vocales ocasionales, los pacientes disfónicos eran más preocupados por los efectos de su problema de voz sobre su carrera futura (el $78 \%$ frente el $24 \%$ ) y el $49 \%$ de ellos afirmaron que su problema de voz tuvo un efecto adverso sobre su trabajo en el pasado comparado con el $4 \%$ de los sujetos "sanos". Tener el problema vocal limita el trabajo actual en el $39 \%$ del grupo de los disfónicos pero solamente en el $2 \%$ del grupo de los "sanos".
Murry y Rosen consideran que es posible identificar la opinión de un paciente sobre la severidad de su trastorno vocal usando una de varias medidas subjetivas específicas de la voz y que usar estas medidas puede dirigir el tratamiento de una manera más específica. Por ejemplo, aunque un paciente puede tener un pólipo 0 un quiste de la cuerda vocal, un índice bajo de discapacidad vocal puede indicar que la cirugía inmediata es innecesaria y puede ser más apropiada una conducta más conservadora. Estos autores afirman que, en ausencia de enfermedad significativa, el grado de discapacidad del paciente y la necesidad percibida de recuperar la función vocal pueden determinar el tipo tratamiento, de esta manera, los recursos y el tiempo se pueden utilizar de una manera apropiada ${ }^{3}$.

En la literatura encontramos diversos estudios que demuestran la importancia de las medidas subjetivas, como el VHI, en la valoración de los trastornos vocales, así:

Rosen y Murry valoraron la utilidad del VHI para determinar la gravedad relativa de los trastornos vocales en tres grupos de pacientes (disfonías funcionales, lesiones benignas de cuerdas vocales y parálisis unilateral de cuerda vocal) antes y después del tratamiento; y concluyen que los pacientes con parálisis de cuerda vocal tienen la más alta percepción de impedimento (VHI más alto), mientras que los pacientes con lesiones benignas de cuerdas vocales tienen la menor percepción de la gravedad (VHI más bajo, cercano a la normalidad) y concluyen que el VHI es un instrumento útil para evaluar el grado de discapacidad en estos trastornos vocales?. 
Los mismos autores en otro trabajo, mediante un estudio caso-control, examinaron el uso de VHI en 106 cantantes (la mayoría con alguna dificultad relacionada de manera específica con la voz cantada, aunque no afectaba a su voz hablada) y 369 pacientes no cantantes con problemas vocales, encontrándose una diferencia significativa en la puntuación entre los dos grupos, e incluso dentro del grupo de los cantantes entre los profesionales y los aficionados. Este estudio señala, también, que la percepción del impedimento de un cantante puede no medirse de manera adecuada visual 0 objetivamente y que el $\mathrm{VHI}$ señala las necesidades específicas y la gravedad de la discapacidad del cantante, aunque la calidad de su voz sólo puede estar levemente alterada ${ }^{9}$.

Hsiung en un estudio sobre 79 pacientes disfónicos con una variedad de trastornos vocales, usando el VHI, encontró que la subescala orgánica era la más afectada en todos los pacientes; y afirma que el VHI proporciona un mecanismo para que el paciente defina su malestar físico, funcional y orgánico; y para que los profesionales de la voz mejoren los programas de tratamiento según las necesidades del paciente ${ }^{10}$.

Este mismo autor, en otro estudio, encontró que cada subescala del VHI proporciona un nivel significativo de la confiabilidad $(p<0,01)$, sin embargo encontró una discrepancia grande entre los resultados de VHI y los resultados de los métodos objetivos del laboratorio de voz lo que demuestra, según el autor, que todavía ningún parámetro objetivo puede considerarse como factor pronóstico definitivo en la evaluación de los pacientes disfónicos ${ }^{11}$.

\section{CONCLUSIÓN}

Concluimos que la presencia de disfonías funcionales supone una discapacidad importante a nivel de las actividades sociales y laborales del paciente y un impacto emocional considerable, reflejado por el índice de VHI y por el índice de QLI; por lo que consideramos que la disponibilidad de programas educativos y la investigación sobre estas patologías es necesaria para mejorar la capacidad funcional de los pacientes y prevenir resultados adversos en su vida.

Este trabajo no fue financiado mediante becas o ningún otro soporte financiero.

\section{BIBLIOGRAFÍA}

1. Sataloff RT, Abaza MM. Impairment, disability, and other medical-legal aspects of dysphonia. Otolaryngol Clin North Am 2000; 33: 1143-52.

2. Benninger MS, Ahuja AS, Gardner G, Grywalski C. Assessing outcomes for dysphonic patients. $J$ Voice 1998; 12, 540-50.

3. Murry T, Rosen C. Medición de resultado y calidad de vida. Clínicas Otorrinolaringológicas de Norteamérica. Ed.: McGraw-Hill Interamericana, 2000; 837-48.

4. Hogikyan ND, Sethuraman G. Validation of an instrument to measure voice-related quality of life (V-RQOL). J Voice 1999; 13, 557-69.

5. Jacobson BH, Johnson A, Grywasky C. The Voice Handicap Index (VHI): development and validation. J Voice 1998; 12, 540-550.

6. Wilson Ja, Deary IJ, Millar A, Mackenzie K. The quality of life impact of dysphonia. Clin Otolaryngol 2002; 27: 179-82.

7. Smith E, Taylor M, Mendoza M, Lemke J, Hoffman $H$. Functional impact of nodules: a casecomparison study. J Voice 1998; 12, 551-8.

8. Verdolini K, Ramig LO. Review: occupational risks for voice problems. Logoped Phoniatr Vocol 2001; 26: 37-46.

9. Rosen CA, Murry T. Voice handicap index in singers. J Voice 2000; 14: 370-7.

10. Hsiung MW, Lu P, Kang BH, Wang HW. Measurement and validation of the voice handicap index in voice-disordered patients in Taiwan. Laryngol Otol 2003; 117: 478-81.

11. Hsiung MW, PaI L, Wang HW. Correlation between voice handicap index and voice laboratory measurements in dysphonic patients. Eur Arch Otorhinolaryngol 2002; 259: 97-9. 